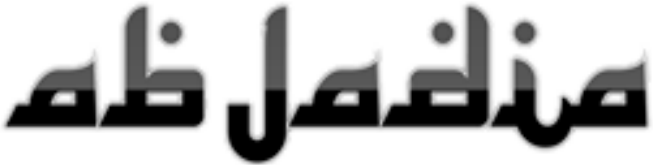 \\ International Journal of Education}

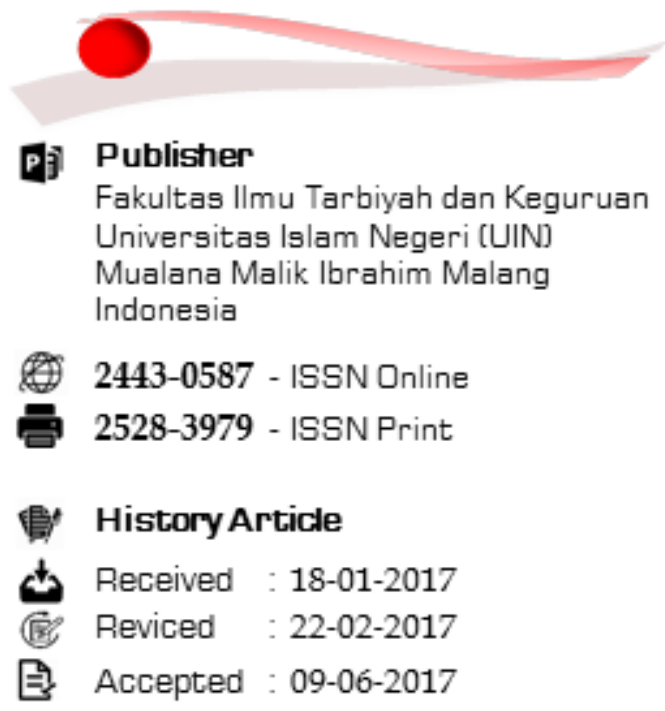

\section{doi.org/ 10.18860/abj.v2i1.5307}

http://ejournal.uin-malang.ac.id/index.php/ abjadia/article/view/5307

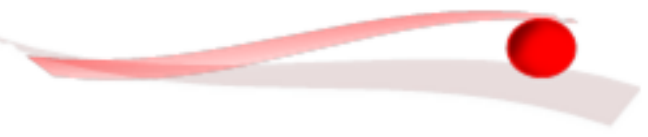

\section{Q Nur Fajar Akbarwari}

I. Indonesia

(3) Universitas Islam Negeri Maulana Malik Ibrahim Malang Indonesia

\section{Corresponding Author}

(C) 081999752496

(9) nur.fajar95@gmail.com

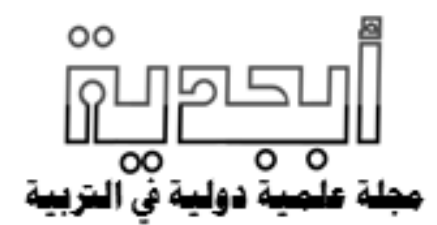

Nur Fajar Akbarwati

Indonesia

$$
\begin{aligned}
& \text { تطوير معجم كتاب تعليم اللغة العربية للصف } \\
& \text { الرابع بالمدرسة الابتدائية }
\end{aligned}
$$

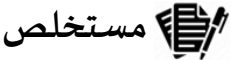

إن تعليم اللغة العربية لا سيما في مدرسة أحمد ياني الإبتدائية

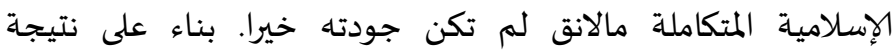

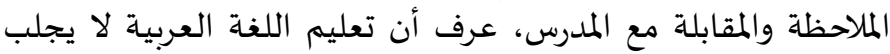

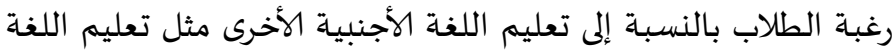

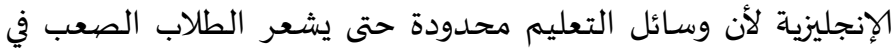

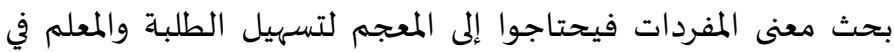

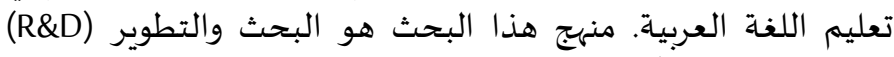

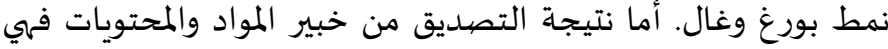

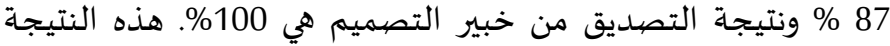

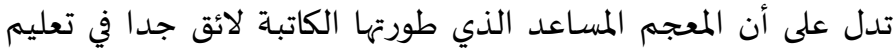

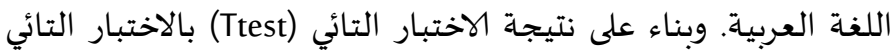

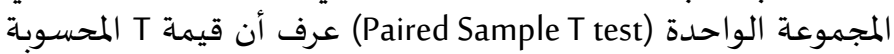

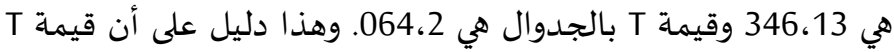

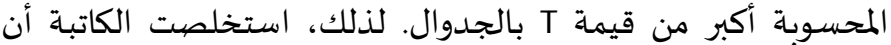

$$
\begin{aligned}
& \text { تعليم اللغة العربية باستخدام المعجم المساعد فعال } \\
& \text { ج (المعتجم، تعليم اللغة العببية ، الملدرسة الإبتدائية }
\end{aligned}
$$

\section{揩" Abstract}

Learning Arabic especially in SDIT Ahmad Yani Malang have not good quality. Based on the observations and interviews with teachers it is known that the students are less fond of Arabic subjects compared with foreign language subjects namely English. Another case is the use of Arabic language learning media especially in the fourth class is still limited so that students feeling it very difficult to know and search the meaning of vocabulary of Arabic material. Therefore needed a supporting dictionary to facilitate teachers and students in the process of learning Arabic as a solution of the problem. This research research and development $(R \& D)$ based on model from Borg and Gall. The result of validation the content and material experts is $87 \%$ and the design expert's validation is $100 \%$. Based on the validation result from both experts, it can be concluded that this supporting dictionary is very suitable for use in the learning Arabic language. Based on the results of Ttest with Paired Sample TTest known THit value is 13.346 and TTabl value is 2, 064. This shows that the value of THit is greater than the value of TTabl it can be concluded that learning Arabic language are effective by using the dictionary support textbook.

\section{@. Dictionary, Learning Arabic, Primary School}




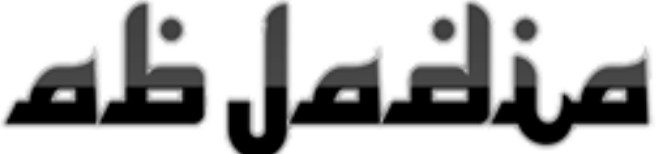
International Journal of Education

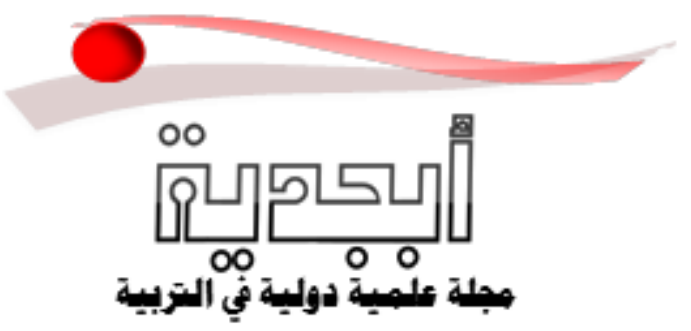

\section{Abstrak}

Pembelajaran bahasa Arab di SDIT Ahmad Yani Malang belum memiliki kualitas baik. Dari hasil observasi dan wawancara, diketahui siswa kurang suka bahasa Arab dibanding pelajaran bahasa asing lainnya seperti bahasa Inggris. Penyebabnya, media pembelajaran bahasa Arab masih terbatas sehingga siswa merasa sulit mengetahui dan mencari arti kosakata. Karena itu dibutuhkan kamus penunjang untuk memudahkan guru dan siswa dalam proses pembelajaran. Metode penelitian ini adalah penelitian dan pengembangan (R\&D) model dari Borg and Gall. Hasil validasi ahli isi dan materi $87 \%$ dan hasil validasi ahli desain $100 \%$. Hasil validasi dari kedua ahli ini menunjukkan bahwa kamus penunjang yang disusun penulis layak dipakai dalam pembelajaran bahasa Arab. Berdasarkan hasil Uji T dengan Paired Sample TTest diketahui nilai, THit 13,346 dan nilai TTabl 2,064. Hal ini menunjukkan bahwa nilai THit lebih besar dari nilai TTabl sehingga kesimpulannya pembelajaran bahasa Arab menjadi lebih efektif dengan kamus penunjang buku ajar di tingkat Madrasah Ibtidaiyah

○. Kamus, Pembelajaran Bahasa Arab, Madrasah Ibtidaiyah

http:// $\quad$ http://ejournal.uin-malang.ac.id/index.php/abjadia/article/view/5307

\section{(C) مقدمة}

الهدف من تعليم اللغة العربية هو تطوير مهارات الطلاب في استعمال اللغة من حيث الكلام

والكتابة بشكل جيد. مهارات استعمال اللغة في تعليم اللغة العربية هي المهارة اللغوية الأربع وهي: الاستماع والكلام والقراءة والكتابة. واللغة العربية لها عناصر وهي الأصيوات والمفردات والتراكب. أما تعليم المفردات

فهي إحدى من عنصر المهم في تعليم اللغة (Mustofa 2011).

كانت المفردات من أهم العناصر اللغوية لأن لها دور كبير جدا ومرتبط بالإتصالية . والشخص الذي

لا يملك عنصر المفردات سيشعر بالصعب في مواصلة هدفه ورغبته وأفكاره مع الأخرين. أما جودة مهارات الشحض اللغوية فتعتمد بكمية المفردات وجودتها. وكثيرا ما عدد المفردات التي يملكها الشخص يمكنه كثيرا في استيعاب المهارة اللغوية. استخدام المفردات المحدودة سواء من جانب كمية وجودة سيكون العائق في قبض وتعبير الأفكار بالمنطقي، والمنتظم، والكامل. لذلك للمفردات دور مهم جداً في قدرة الطفل أو الطالب أن يقبض ويفهم الفكر من المتكلم لترقية جودة التعليم. ويجب أن يكون الغرض الرئيسي من تعليم المفردات تنمية رغبة الطلاب على الكلمة. والطالب الذي يرغب أن يعرف اللغة سيكون شاعرا بالسهولة لإثراء المفردات والتمييز والتفكير المنطقي (Nurhadi 1995). 
كان تعليم المفردات محدود بسبب نقص المتنوعات على الوسائل التعليمياة. وتزويد القدرة توكيدها في اللغة العربية يحتاج إلى الأدوات المسـاعدة الأخرى مثل المعجم. فيكون المعجم من أنواع الكتب التعليمية التي تساعد عملية التعليم. والمعجم أو القاموس عند أحمد عبد الغفور عطار هو كتاب يضم أكبر عدد من مفردات اللغة مقرونة بشرحها وتفسير معانها على أن تكون المواد مرتبة ترتيبا خاصيا، إما على حروف الهجاء أو الموضوع (Taufiqurrochman 2008). وفي مدرسـة أحمد ياني الابتدائية الإسلامية المتكاملة مالانق للصف الرابع هناك يُستخدم كتاب تعليم اللغة العربية بالعنوان "Kreatif Belajar Bahasa Arab" بالمنهج الدراسي 2013. وكثيرا ما من المشكلات التي واجهها الطلاب في تعليم اللغة العربية قلة الأدوات أو الوسائل التي تساعد المعلم والطلاب حتى تجعل الطلاب بطيءا ومملّا في اشتراك الدرس. وهناك لم يوجد المعجم المساعد على كتاب تعليم اللغة العربية للصف الرابع.

ليس نادر الوقوع أن تعليم اللغة العربية في مدرسـة أحمد ياني الابتدائية الإسلامية المتكاملة مالانق لا يجلب رغبة الطلاب بالنسبة إلى تعليم اللغة الأجنبية الأخرى وهي اللغة الإنجليزية. لأنهم يشعرون الصعب في بحث ومعرفة معنى المفردات من مادة اللغة العربية (مقابلة مع مفلخون يشريف، مدرس اللغة العربية للصف الرابع، تاريخ 19 يوليو 2017). وهذا يؤثر في ضعف فهم الطلاب على مادة اللغة العربية. وإن كان التعليم الجيد غير مجرد تبليغ الكلمة ولكن الحاجة إلى اكمال الأدوات أو الوسائل مهم جدا تسهيلا للطلاب في فهم الدرس. ولذلك، بناء على هذه المشكلة، تريد الكاتبة تطوير المعجم المسـاعد على كتاب تعليم اللغة العربية للصف الرابع في مدرسة أحمد ياني الابتدائية الإسلامية المتكاملة مالانق لتسهيل الطلاب والمعلم في التعليم اللغة العربية.

\section{[] منهج}

\section{مدخل البحث ومنهجهـ}

منهج هذا البحث هو منهج البحث والتطوير Research and Development (R\&D) وهو طريقة البحث المستخدم للحصول على إنتاج معين وتجربة فعالية (Sugiyono 2015). هذا البحث يستخدم نمودج البحث والتطوير من بورك وغال (Borg and Gall). مدخل هذا البحث هو المدخل الكيفي والكمي. أما المدخل الكيفي فهو يعرض ويبين كيفية تطوير المعجم المساعد على كتاب تعليم اللغة العربية للصف 
الرابع في مدرسة أحمد ياني الابتدائية الإسلامية المتكاملة مالانق. أما المدخل الكمي فيفيد إلى معرفة مقياس فعالية المعجم المطور في التجربة.

$$
\text { أدوات البحث }
$$

أدوات البحث المستخدمة في هذه البحث: 1) الملاحظة 2) المقابلة 3) الوثائق 4 ) الاستبانة 5)

الاختيار.

أسلوب تحليل البيانات

أن تحليل البيانات دور مهما في البحث العلمي بالتحليل تكون البيانات ذو معاني كبيرة. وتستخدم

الباحثة التحليل الوصفي الكيفي والتحليل الإحصائى الوصفي لتحليل البيانات في هذا البحث كالآتي:

تحليل الوصفي الكيفي (Descriptive Analysis Qualitative)

تحلل الباحثة البيانات الكيفية هي البيانات من الوثائق، المقابلة والبيانات عن تعليقات ومقترحات

من الخباراء، الاستبانة من المدرس والطلاب بالأسلوب الوصفي الكيفي.

تحليل الإحصيائى الوصفي (Descriptive Analysis Statistic)

تحلل الباحثة البيانات الكمية هي البيانات عن تصيديق خبراء واستبانة من المدرس والطلاب بالأسلوب تحليل الإحصائى الوصفي لمعرفة نجاح أو اللياقة هذا الإنتاج أي المعجم المساعد أن تطبيف في تعليم اللغة العربية.

تحليل الاختبار التائي المجبموعة الواحلدة (Paired Sample T test)

تحلل الباحثة البيانات المحصولة من الاختبار قبلي وبعدي، وتقوم الباحثة بالاختبار التائي المجموعة الواحدة (Paired Sample T test) . يتم إجراء هذا الاختبار على اثنين من العينة المقترنة. المراد بالعينة المقترنة هي عينة بالفاعل سواء ولكن يجرب الإجرائتين أو قياسـة مختلفة. ومثل الشخص A سوف يجد إجراءة الأول ثم إجراءة الثاني (Santoso 2010). تستخدم الباحثة معيار المعنوية (0،05/ 0,05) والرموز

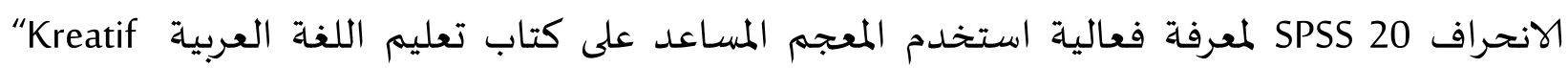
باعتبار نتائج الاختبار قبلي وبعدي. بعد معرفة نتائج اختبار الإختلاف فسيجد أن Belajar Bahasa Arab" يثبت الفروض في هذا البحث كالآتي: 
Ho : إن استخدم المعجم المسـاعد على كتاب تعليم اللغة العربية للصف الرابع في مدرسة أحمد ياني الابتدائية الإسلامية المتكاملة مالانق غير فعالية. إن استخدم المعجم المساعد على كتاب تعليم اللغة العربية للصف الرابع في مدرسة أحمد : Hi ياني الابتدائية الإسلامية المتكاملة مالانق فعالية.

\section{نتائج مفهوم الكتاب التعليمي} أن الكتاب التعليمي عنصرا أسـاسيا في عملية التعليم والتعلّم لتحقيق الأهداف التي تريد تحقيقها، بالإضـافة إلى المكونات الأخرى من أنشطة وطرق التدريس (جعفر 2007). والكتاب التعليمي احدى مصادر التعليم كالتعلّم الذي يساعد على النجاح عملية التعليمياة. ويساعد الكتاب التعليمي مساعدة كبيرة على الأساتيذ والطلبة في عملية التعليم كالتعلّم خصيوصا في تعليم اللغة العربية. أهمية الكتاب التعليمي وإذا كان الكتاب التعليمي لله أهميته في العملية التعليمية فهو أحد مكوناتها وله فوائده للدارس كالمعلم، فهو من جانب آخر له أضراره ومساوئه على الدارس واتجاهاته ومن ثم فهو سلاح ذو حدين، بقدر ما يفيد، فهو أحيانا قد يضر إذا لم يعد إعداد جيدا وإذا لم يتم اختياره وفق مبادئ وأسس منشودة، ووفق أهداف ينشـدها المجتمع والذين الإسلامي، ومن هنا تظهر قيمة إعداد الكتب التعليمية، خاصية لتعليم العربية للناطقين بغيرها (وأخرون 1992).

\section{مفهوم المعجم ووظيفته}

المعجم أو القاموس هو كتاب يحتوي على كلمات منتقاة، ترتيب عادة ترتيبا هجائيا، مع شرح لمعانيها

ومعلومات أخرى ذات علاقة بها، سواء أعطيت تلك الشروح والمعلومات باللغة ذاتها أم بلغة أخرى (على 1991). هناك مجموعة من الوظائف يجب أن يؤديها المعجم وهي: 1) شرح الكلمات وبيان ومعنيها، إما في العصر الحديث فقط أومع تتبع معانها أو معانها عبر العصور. 2) بيان كيفية نطق الكلمة. 3) بيان كيفية كتابة الكلمة. 4) تحديد الوظيفة الصرفية للكلمة. 5) بيان درجة اللفظ في الاستعمال، ومستواه في سلم التنوعات اللهجية. 6) حديد مكان النير في الكلمة. والنير باختصر هو إعطاء برموز معين لأحد مقاطع الكلمة دون المقاطع الأخرى (مختار 1988). 
رأي شهاب الدين هناك أربعة شروط يجب الوفاء بها من أجل جعل المعجم المثالي، المعجم هو جيد ويفي بمعايير تماما، أربعة معايير هي معجم المثال: 1) الكمال 2) الإيجاز 3) الدقة 4) سهولة الشرح (مختار 1988).

أنواع المعجم

\begin{tabular}{|c|c|}
\hline 2. من ناحية التأليف & 1. من ناحية الموضوع \\
\hline ب) أ) الموضيائى & ب) \\
\hline 4. من ناحية عدد اللغة المستخدمة & 3. من ناحية المادة \\
\hline 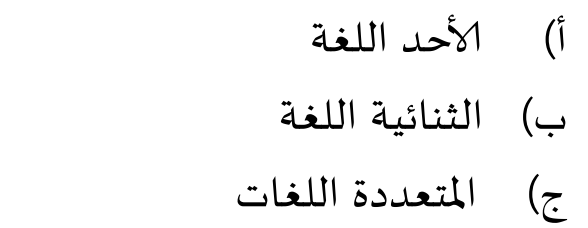 & ب) ال الخامة \\
\hline
\end{tabular}

مفهوم المدرسة الابتدائية

المدرسـة الابتدائية هي المرحلة الأولى التي يدخل إليها الطلاب للتعلّم، وهي مرحلة إلزاميّة، حيث يجب على كافة الطلاب ومن مختلف الطبقات الاجتماعية أو الاقتصادية الالتحاق بها، وتتكون عادةً من خمسة إلى ستاة صفوف، حسب الدولة، كما وتعتبر من أهم المراحل في حياة الطلاب (بطمة، إيمان 2017).

أهداف التعليم في المرحلة الابتدائية

أمّا أهداف التعليم في المرحلة الابتدائية كما يلي: 1) تثبيت العقيدة الإسلامية وتربية الطالب وتربية إسلامية قوامها القرآن نصا وروحا في خلقاه وجسماه وعقله 2) تنمية المهارات الأساسية المختلفة وخاصة المهارات اللغوية 3) تزويد الطالب بالقدر المناسب من المعلومات في مختلف الموضوعات 4) تعريف الطالب بنعم الله على نفسـاه وبدناه وبيئته الجغرافية والاجتماعية 5) تنمية العمل اليدوي لله ورفع شأنه بما يقدمه لنفسـه والآخرين 6) تعريف الطالب ما عليه من حقوق وواجبات وفق سنّه وغرس حب الوالدين وما حوله في نفسـ 7) توليد الرغبة لدياه في حب العلم والعمل الصالح، والاستعداد للمراحل القادمة في حياته (بطمة إيمان 2017). أهمية المدرسـة الابتدائية 
أمّا أهمية المدرسة الابتدائية فكما يلي: 1) هي المرحلة الأولى والأساسيّة التي يدخلها الطلاب لتلقي تعليمهم، وفيها يبدؤون بتعلّم أصول القراءة والكتابة الصحيحة 2) تبدأ في هذه المدرسـة عمليّة تكوين شخصيّة الطلاب 3) الكشف عن الجانب الفكري والمعلوماتي للطلاب، بالإضافة إلى تنمية مهاراتهم المختلفة 4) تعتبر المدرسة الابتدائية هي المكان الذي يبدأ فيه الطلاب ببناء هوياتهم الوطنيّة 5) تعتبر مرحلة البناء العاطفي للأطفال 6) معرفة الطلاب المعنى الصحيح للحقوق والواجبات، بالإضافة إلى تعلّم النظام، والالتزام بالمواعيد والأحكام المفروضة في المدرسة (بطمة إيمان 2017).

\section{مُبْز}

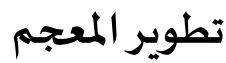

في هذا البحث استحدام المنهج التطويري (R\&D) ونمودج التطويري بورغ وغال تتكون من عشرة

مراحل. ولكن في عملية تطوير هذا المعجم المساعد على كتاب تعليم اللغة العربية Kreatif Belajar استخدمت الباحثة بتسعة مراحل. لأنه عملية تطوير هذا المعجم المساعد يوفق على الأحوال في الميدان وممكن أن ينفذ بتسعة مراحل أي دون ينفذ إصلاح الإنتاج الثالث من البيان كالآتي : (1) تحليل الحاجة والمشكلات (2) جمع البيانات (3) تصميم المعجم (4) (5) إصلاح الإنتاج الأولى إلى (6) تجربة الإنتاج الأول (7) إصلاح الإنتاج الثاني (8) تجربة الميدانية (9) الإنتاج الجماعي. فعالية استخدام المعجم المساعد

(T أما تحلل الباحثة البيانات المحصولة من الاختبار قبلي وبعدي، وتقوم الباحثة بالاختبار التائي (test) وتقوم الباحثة بالاختبار التائي المجموعاة الواحدة أو (Paired Sample T test) . أدخلت الباحثة هذه النتائج الاختبار القبلي والبعدي من خمسـة وعشرون طالبا وعرضت الباحثة نتائج الاختبار القبلي والبعدي لمعرفة فعالية استخدم هذا المعجم المساعد. واستخدمت الباحثة رموز الانحراف SPSS 20 في معرفة فعالية استخدم المعجم المساعد على كتاب "Kreatif Belajar Bahasa Arab". ونتائجها كالآتي:

\begin{tabular}{|ll|c|c|c|c|}
\hline & Mean & N & Std. Deviation & $\begin{array}{c}\text { Std. Error } \\
\text { Mean }\end{array}$ \\
\hline \multirow{2}{*}{ Pair 1 } & POST TEST & 67,80 & 25 & 9,251 & 1,850 \\
& PRE TEST & 89,00 & 25 & 9,789 & 1,958 \\
\hline
\end{tabular}


نظر إلى الجدوال يدل نتيجة متوسط من الاختبار القبلي والاختبار البعدي. وجدت الباحثة الفرق نتيجة متوسط من الاختبار القبلي هو 67،80 ونتيجة متوسط من الاختبار البعدي هو 89،00. فتقرر الباحثة أن موجود الارتفاع على نتيجة التعليمية طالبا بعد استخدام المعجم المساعد في تعليم اللغة العربية.

\begin{tabular}{|ll|c|c|c|}
\hline & $\mathbf{N}$ & Correlation & Sig. \\
\hline Pair 1 & POST TEST \&PRE TEST & 25 &, 653 &, 000 \\
\hline
\end{tabular}

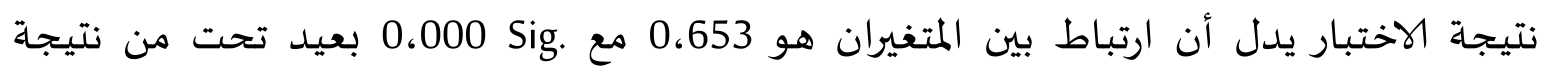
احتمالية أي 05،0. نظر إلى الجدوال نتائج ارتباط المتغيران يدل أن ارتباط بين المتغيران من الاختبار القبلي والاختبار البعدي هو قوة ومعنوي.

\begin{tabular}{|c|c|c|c|c|c|c|c|c|}
\hline & \multicolumn{5}{|c|}{ Paired Differences } & \multirow{3}{*}{$\mathbf{t}$} & \multirow{3}{*}{ df } & \multirow{3}{*}{$\begin{array}{c}\text { Sig. } \\
\text { (2-tailed) }\end{array}$} \\
\hline & \multirow[t]{2}{*}{ Mean } & \multirow[t]{2}{*}{$\begin{array}{c}\text { Std. } \\
\text { Deviation }\end{array}$} & \multirow{2}{*}{$\begin{array}{l}\text { Std. } \\
\text { Error } \\
\text { Mean }\end{array}$} & \multicolumn{2}{|c|}{$\begin{array}{c}\text { 95\% Confidence } \\
\text { Interval of the } \\
\text { Difference }\end{array}$} & & & \\
\hline & & & & Lower & Upper & & & \\
\hline $\begin{array}{c}\text { Pair } 1 \\
\text { POST TEST } \\
\text { PRE TEST }\end{array}$ & $-21,200$ & 7,943 & 1,589 & $-24,479$ & $-17,921$ & $-13,346$ & 24 & ,000 \\
\hline
\end{tabular}

بناء على الجدوال نتائج الاختبار التاء (T) معرفة أن قيمة T المحسوبة هي 13، 1346 وأما قيمة بالجدوال هي 2،64، وهذا دليل أن قيمة T المحسوبة أكبر من قيمة T بالجدوال وهذا يدل على أن تعليم اللغة العربية باستخدام المعجم المساعد فعال. وأيضيا 050، Sig وأما قيمة .000 Sig أصغر من 05،0 وهذا الاختبار دليل أن موجود الفرق بالمعنوي بين نتيجـة الاختبار القبلي والاختبار البعدي.

\section{国}

تطوير المعجم المساعد على كتاب تعليم اللغة العربية للصف الرابع في مدرسة أحمد ياني الابتدائية الإسلامية المتكاملة مالانق تمر تسعة مراحل هو كما يلي: (1) تحليل الحاجة والمشكلات، يقوم بالمقابلة مع مدرس اللغة العربية وتحليل الحاجة بالاستبانة للطلاب الفصل الرابع. (2) جمع البيانات، تبدأ الباحثة أن تجمع المعلومات أو البيانات من كتاب التعليمي "Kreatif Belajar Bahasa Arab" للصف الرابع. (3) تصيميم المعجم، ترتيب الباحثة المفردات وتكتب جميع المفردات مكتوبة داخل الجدول فالباحثة ترتيب 
المفردات حسب الألفبائي ببرنمج Microsoft Word باستخدام تطبيق الحاسوب يسمى بتطبيق Adobe Photoshop CS3 وتطبيق Power Point ومقياس القرطاس A5 بالجنس Glory. (4) تصيديق من الخبراء، نتيجة تصيديق من الخبير المواد والمحتويات هي 87 \% ونتيجة تصديق الخبير التصيميم هو 100\%. (5) إصلاح الإنتاج الأولى، وأصلحت الباحثة الإنتاج باعتماد على اقترحات من الخبيرين قبل التجربة الأولى. (6) تجربة الإنتاج الأول، فالباحثة يقوم بتجربة الإنتاج الأولى أو المحدودة إلى المدرس اللغة العربية هو أستاذ مفلخون وست طلاب في الفصل الرابع بوسيلة الاستبانة محدودة. (7) إصلاح الإنتاج الثاني، أصلحت الباحثة الإنتاج باعتماد على اقترحات من ست الطلاب ومدرس اللغة العربية. (8) تجربة الميدانية، أخذت الباحثة بيانات الاستبانة إلى خمسة وعشرون طالبا. (9) الإنتاج الجماعي، وهذه هي الخطوة الأخيرة بعد انتهاء الخطوات العديدة فالباحثة تطبع الإنتاج في الطبعة.

بناء على الاختبار التائي (T test) بالاختبار التائي المجموعة الواحدة أو (Paired Sample T test)

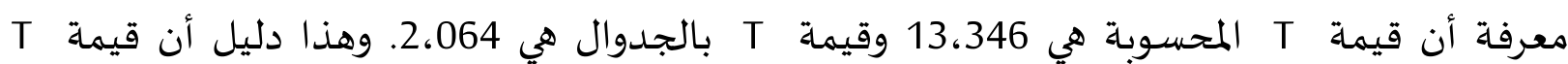
المحسوبة أكبر من قيمة T بالجدوال. فاستننجت الباحثة أن تعليم اللغة العربية باستخدام المعجم المساعد على كتاب تعليم اللغة العربية للصف الرابع في مدرسة أحمد ياني الابتدائية الإسلامية المتكاملة مالانق فعالية.

تقدمت الباحثة التوصيات كالآتي: 1) ترجو الباحثة المدرسة أن يستخدم هذا المعجم خاصة للمدرس والطلاب في المرحلة الإبتدائية لأن هذا المعجم مهم جدا لتسهيل عملية تعليم اللغة العربية. 2) ترجو الباحثة المدرس أن يستخدام وسـائل التعليمية المتنوعات وابتكار يستخدام وسـائل التعليمية المناسبة بحاجة الطلاب في تعليم اللغة العربية.

ومن الواضح أن هذا البحث محدود تطوير المعجم المساعد على كتاب تعليم اللغة العربية بالعنوان "Kreatif Belajar Bahasa Arab" للصف في مدرسة أحمد ياني الابتدائية الإسلامية المتكاملة مالانق. وتصنيف الباحثة المعجم بموضوع معين وترتيب المفردات بالألفبائي وقليل زيادة الصهور. تقدمت الباحثة المقترحات للباحثين المستقبل أن موجود عدد الموضوع البحث تستطيع أن يبحث مثل المعجم السياقي ثلاثة اللغة بالصيور أو المعجم المساعد الخاص يفرق بين الأفعال والاسماء بالصور أو مستوى المدرسة الآخر أو يمكن بصناعة المعجم من كتاب الآخر وغير ذلك. وللباحثين المستقبل أن يعد معجم الطلاب أحسن وأكمل وإبتكار من هذا المعجم باستخدام أكثر المراجع أو المفردات. 
Mustofa, Syaiful. 2011. Strategi Pembelajaran Bahasa Arab Inovatif. Malang: UIN Malang Press.

Nurhadi. 1995. Tata Bahasa Pendidikan: Landasan Penyusunan Buku Pelajaran Bahasa.

Semarang: IKIP Semarang Press.

Santoso, Singgih. 2010. Mastering SPSS 1. Jakarta: PT Elex Media Komputindo.

Sugiyono. 2015. Metode Penelitian Pendidikan: Pendekatan Kuantitatif, Kualitatif, Dan RED. Bandung: Alfabeta.

Taufiqurrochman, R. 2008. Leksikologi Bahasa Arab. Malang: UIN Malang Press.

$$
\begin{aligned}
& \text { إيمان, بطمة. 2017. "شبكة أهداف التعليم في المرحلة الابتدائية."شبكة مفهوم الملمربسة للبتدائية . } \\
& \text { Retrieved (http://mawdoo3.com). } \\
& \text { إيمان, بطمة،، 2017. “مفهوم المدرسة الابتدائية."شبكة مفهوم الملدرسة للبتدائية Retrieved. } \\
& \text { (http://mawdoo3.com). } \\
& \text { جعفر, الخلفية، حسن. 2007. مدخل إلى المناهج وطرق التدريس. بيروت: مكتبة الرشد ناشرون. }
\end{aligned}
$$

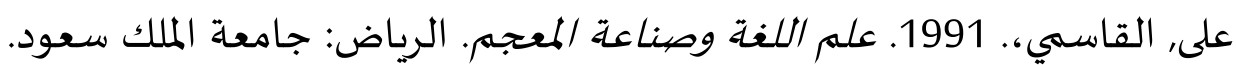

$$
\begin{aligned}
& \text { مختار, عمر، أحمد. 1988. البحث اللغنوى عند العرب. القاهرة: علم الكتب. } \\
& \text { وأخرون, رضوان، أبو الفتح. 1992. الكتاب الملدرسي. القاهرة: مكتبة الأنجلو المصرية. }
\end{aligned}
$$

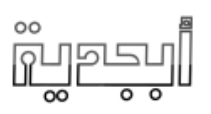

